\title{
The Security of Chaffing and Winnowing
}

\author{
Mihir Bellare and Alexandra Boldyreva \\ Dept. of Computer Science \& Engineering, University of California at San Diego, \\ 9500 Gilman Drive, La Jolla, California 92093, USA. \\ \{mihir, aboldyre\}ecs.ucsd.edu \\ www-cse.ucsd.edu/users/\{mihir, aboldyre\}
}

\begin{abstract}
This paper takes a closer look at Rivest's chaffing-and-winnowing paradigm for data privacy. We begin with a definition which enables one to clearly determine whether a given scheme qualifies as "chaffing-and-winnowing." We then analyze Rivest's schemes to see what quality of data privacy they provide. His bit-by-bit scheme is easily proven secure but is inefficient. His more efficient scheme — based on all-or-nothing transforms (AONTs) — can be attacked under Rivest's definition of security of an AONT, and even under stronger notions does not appear provable. However we show that by using OAEP as the AONT one can prove security, and also present a different scheme, still using AONTs, that is equally efficient and easily proven secure even under a relatively weak notion of security of AONTs.
\end{abstract}

\section{Introduction}

Rivest presents a number of methods to achieve data privacy based on a paradigm he calls "chaffing and winnowing" [11]. In this paper we provide a definition of chaffing and winnowing; assess whether the schemes of [11] can be proven to meet standard data-privacy goals, and, if so, under what kinds of assumptions on the underlying primitives; and suggest more efficient schemes and analyze their security. Let us first provide some background and motivation, and see what are the basic questions. Then we discuss our contributions in more detail.

\subsection{Background, Motivation, and Questions}

Chaffing and winnowing uses a message authentication code (MAC) to provide privacy. However Rivest notes that in order to have privacy the MAC must be a pseudorandom function. (Any PRF is a good MAC [9.3] but not vice-versa.) Of course, there are many well-known ways to use a PRF to provide privacy; the interest of chaffing and winnowing arises from the particular manner in which the MAC is used, which is roughly the following. Each data block is authenticated so that one has a sequence of data-MAC pairs. Then "chaff" is interspaced, this consisting of pairs, each being a block with a random tag. The receiver can discard blocks with invalid tags - this is called "winnowing"- thereby recovering the data. (Within this framework, many specific methods are possible.) Privacy requires that it be computationally infeasible for an adversary to tell valid MACs from random tags. (But is also very sensitive to the manner in which chaff is interspaced.) Rivest argues that the use of the MAC here stays within

T. Okamoto (Ed.): ASIACRYPT 2000, LNCS 1976, pp. 517-530 2000.

(C) Springer-Verlag Berlin Heidelberg 2000 
its functionality as an authentication mechanism, and thereby makes moot a policy that restricts "encryption" while allowing authentication.

Chaffing-and-winnowing has received a lot of attention on the political front, but little on the technical front barring that in the initial paper. It merits more serious attention from cryptographers. One is that a better technical understanding leads to a better understanding of the implications for the debate on cryptographic policy. Another reason is foundational. As Rivest notes in introducing his idea, there are very few paradigms for cryptography's main goal, namely data privacy. A new paradigm such as the one he presents should be explored in order to assess its potential.

The description and examples in [11] suffice to get the gist of the idea, but as we consider more complex mechanisms it is sometimes difficult to decide whether they obey the "rules of the game." A definition is needed to settle such questions, and also for rigorous security analysis. Accordingly, we begin there.

Chaffing and winnowing purports to provide data-privacy. A basic question is about the quality of privacy that it can provide. Specifically we want to know how it compares to standard mechanisms such as modes of operation of a block cipher, which have been proven to meet strong, well-defined notions of privacy under appropriate assumptions [2]. Can chaffing-and-winnowing based schemes provide the same level of privacy, and, if so, can this be proven, and under what assumptions?

\subsection{Defining Chaffing and Winnowing}

The security goal of a chaffing-and-winnowing scheme is to provide privacy in a symmetric setting. Accordingly, from the security point of view, it is - in fact, must betreated simply as a symmetric encryption scheme. There is some "encryption" process that takes a message and creates a "ciphertext", and some "decryption" process that takes the ciphertext and recovers the message, both operating under a common secret key. (This is the key for the MAC.) These processes are not implemented in "usual" ways, but, abstractly, they must exist, else it is moot to talk of achieving privacy. Once this is understood, security can be measured using any of several well-known notions in the literature. (We adopt the simplest, namely the "find-then-guess" notion of [2], which is the most direct extension to the symmetric case of the notion of indistinguishability of [10].)

Thus what defines chaffing and winnowing as a "notion" is not some novel security property but rather a novel set of restrictions on the processes (namely encryption and decryption) directed at achieving a standard security property (namely data privacy). The crux of the definition is to pin down these restrictions. We view a chaffingand-winnowing based encryption scheme as arising by the use of an authentication to privacy transform (ATPT) over a MAC-based authentication channel.

The channel captures the manner in which the parties have access to the MAC function $\operatorname{MAC}(K, \cdot)$. An application on the sender side can pass data down to be MACed, thereby creating a packet (data-MAC pair) which is transmitted over the channel. (The application has no direct access to the $\operatorname{MAC}(K, \cdot)$ function let alone to the underlying key $K$.) At the receiving end, packets with invalid MACs are dropped and the data from valid packets is passed up to the receiving application. (The latter sees no MACs 
and does not even know of the existence of the dropped packets.) See Figure 1 and Definition 4

The ATPT consists of three algorithms whose important feature is that they are all entirely keyless. The sending application applies a MakeWheat algorithm to the plaintext to turn it into a sequence of data blocks to be passed to the authentication channel. An AddChaff procedure is responsible for interspacing chaff packets into the stream of valid packets output by the authentication channel. Finally the receiving application applies a Recover algorithm to the received data blocks to get back the plaintext. See Definition 5

We stress again that the algorithms in the ATPT are keyless, so that on their own they cannot be used to provide privacy. The chaff and winnow based encryption scheme is realized by coupling these algorithms with the authentication channel. This is illustrated in Figure 2.

The definition (provided in Section 3) can help clarify the contribution of chaffing and winnowing to the debate on cryptographic policy by providing a means to evaluate whether a particular method qualifies as "legal encryption based on authentication." If one scheme meeting the definition qualifies, so do the rest, even if their implementation is more complex.

\subsection{Security of Rivest's Schemes}

Rivest notes that his first few examples will not provide a high level of privacy. (In particular they will not meet a notion of privacy such as find-then-guess.) The first serious candidate is the bit-by-bit scheme.

BIT-BY-BIT SCHEME. Here the MakeWheat procedure splits the plaintext into bits and appends a counter or nonce to each bit. These data blocks are MACed. The AddChaff procedure inserts, for every valid packet, an invalid packet with the opposite bit value and an appended nonce, together with a random value for the tag. We prove that this scheme provides privacy in the find-then-guess sense assuming the MAC is a pseudorandom function. The concrete security analysis is provided in Theorem 1 .

This indicates that chaffing and winnowing can provide privacy of as high a quality as standard encryption schemes, and furthermore with provable guarantees based on the same assumption - namely a pseudorandom function - used to prove the security of popular block cipher modes of operation [2]. There is however a high cost in bandwidth: two nonces and two tags are needed per bit of plaintext.

SCATTERING SCHEMES. In order to reduce the bandwidth, Rivest suggests an alternative paradigm. First apply an all-or-nothing transform (AONT) [12] to the plaintext. (This is a keyless, invertible transform with the property that inversion is hard if any block of the output is missing.) Each block of the output of the AONT is MACed, resulting in a stream of valid packets. Then $s^{\prime}$ chaff packets are inserted into random positions in this stream. Intuitively, an adversary must guess the positions of all $s^{\prime}$ chaff packets in order to decipher. (Accordingly it is suggested that security will be provided for a value of $s^{\prime}$ that does not depend on the length of the plaintext, eg. $s^{\prime}=128$, so this method is cost-effective for long plaintexts.) Upon closer examination, however, the security provided by this paradigm is unclear. We note first that under the original 
definition of an AONT provided in [12] the scheme is insecure. (We show that there are example AONTs that meet the definition of [12] but for which there are attacks compromising the privacy of the chaffing-and-winnowing scheme.) It is natural to then try to use Boyko's stronger definition of security for an AONT [6]. In that case the analysis is inconclusive: the stronger property of an AONT still does not appear to suffice to prove security of the chaffing-and -winnowing scheme, but neither do we exhibit a counter-example that confirms this. We would prefer a provably-secure scheme.

SCATTERING WITH OAEP. The above-mentioned analyses indicate that in general an AONT seems neither necessary nor sufficient as the initial transform to provide privacy of the scattering based chaffing-and-winnowing scheme. We show however that if the OAEP transform of [5] is used as the AONT, then privacy can be proved. (This, like all security proofs involving OAEP, is in a random oracle model [4]). The concrete security analysis provided in Theorem 2 supports the intuition regarding the scattering scheme provided in [11] - the probability of breaking the scheme is inversely proportional to $\left(\begin{array}{c}s+s^{\prime} \\ s\end{array}\right)$ where $s$ is the number of blocks in the output of OAEP and $s^{\prime}$ is the number of chaff blocks.

Note that OAEP has been shown to be a secure AONT [6], but given the above we cannot exploit this here. Instead, our proof is direct, based on techniques from [5.6]. It is an open question whether other specific constructions of AONTs such as that of [7] suffice to prove security of the scheme.

\subsection{New Schemes}

We point out that there is an alternative to the scattering scheme that is simpler, can be proven secure, and is equally cost-effective. It too makes use of AONTs and can be proven secure for any AONT meeting a notion of security that is actually weaker than that of [6]. (In particular one can use OAEP or use the construct of [7] and avoid random oracles but there may be other more efficient instantiations.) The construction applies the AONT to the plaintext as before. Rather than scattering chaff into the output blocks, however, it simply treats a prefix of this output as the plaintext for the bit-by-bit chaffing-and-winnowing scheme and applies the latter. Theorem 3 provides a concrete security analysis of the final chaffing-and-winnowing scheme.

\subsection{Is Chaffing and Winnowing "Encryption"?}

We view chaffing-and-winnowing schemes as (special kinds of) symmetric encryption schemes, the key for encryption and decryption being that of the MAC function. This might at first seem to contradict Rivest's view [11]. He says that the process of chaffing and winnowing is "not encryption" and that there is no "decryption key." These views are not at odds with each other; the difference is purely in terminology. We are using the technical terminology of cryptographers which is more suited to security analysis, while Rivest uses the terminology of cryptographic policy discussion. (The convention in modern cryptography, which we are following here, is to use the term "encryption scheme" for any mechanism whose goal is to provide privacy. Under this convention, the key for the MAC is, by definition, a decryption key, since it enables recovery of the plaintext from the ciphertext. In cryptographic policy, "encryption" seems to refer 
to certain mechanisms rather than a goal. Actually, exactly what it refers to is unclear, which is part of the point made in [11].)

\section{Symmetric Encryption, PRFs, and AONTs}

SYMMETRIC ENCRYPTION. A symmetric encryption scheme $\mathrm{SE}=(\mathcal{K}, \mathcal{E}, \mathcal{D})$ consists of a (randomized) key generation algorithm $\mathcal{K}$ (returning a key $K$ ), a (randomized or stateful) encryption algorithm $\mathcal{E}$ (taking $K$ and a message $M \in\{0,1\}^{*}$ to return a ciphertext $C$ ) and a decryption algorithm $\mathcal{D}$ (taking $K$ and a ciphertext and returning a message). We require that $\mathcal{D}_{K}\left(\mathcal{E}_{K}(M)\right)=M$ for all $M \in\{0,1\}^{*}$. In the "find-thenguess" model [2] an adversary is given an oracle for encryption under key $K$ and wins if it can find two equal-length messages whose ciphertexts it can later distinguish. Below we associate to any adversary an "advantage" which measures its winning probability, and then use as security measure of the scheme the maximum possible advantage subject to stated resource restrictions on the adversary.

Definition 1. (Find-then-guess security of encryption, [2]) Let $\mathrm{SE}=(\mathcal{K}, \mathcal{E}, \mathcal{D})$ be a symmetric encryption scheme. For an adversary $A$ and $b=0,1$ define the experiment

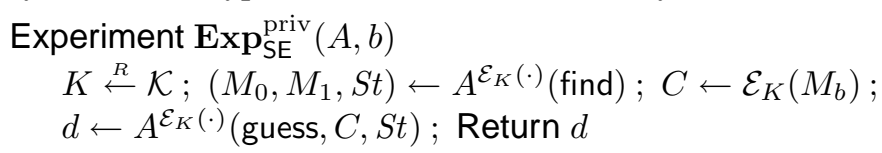

Here $S t$ is some state information that the adversary may want to preserve to help it later. It is mandated that $\left|M_{0}\right|=\left|M_{1}\right|$ above. Now define the advantage of $A$ and the advantage function of the scheme respectively, as follows:

$$
\begin{aligned}
\mathbf{A d v}_{\mathrm{SE}}^{\text {priv }}(A) & =\operatorname{Pr}\left[\mathbf{E x p}_{\mathrm{SE}}^{\text {priv }}(A, 0)=0\right]-\operatorname{Pr}\left[\mathbf{E x p}_{\mathrm{SE}}^{\text {priv }}(A, 1)=0\right] \\
\mathbf{A d v}_{\mathrm{SE}}^{\text {priv }}(t, q, \mu) & =\max \left\{\mathbf{A d v}_{\mathrm{SE}}^{\text {priv }}(A)\right\}
\end{aligned}
$$

where the maximum is over all $A$ with "time-complexity" $t$, making at most $q$ encryption oracle queries, these totalling at most $\mu$ bits.

In this paper for simplicity we assume that all messages encrypted have the same length, usually denoted $m$. This means that $\mu=m q$. We also assume that the length of each of the challenge messages is $m$. The "time-complexity" refers to the worst case execution time of experiment $\operatorname{Exp}_{\mathrm{SE}}^{\text {priv }}(A)$ plus the size of the code of $A$, in some fixed RAM model of computation. We are considering only chosen-plaintext attacks, not chosenciphertext attacks.

PsEudorandom FUnCTIONS. Consider a map $F:\{0,1\}^{k} \times S \rightarrow\{0,1\}^{l}$ which takes a key $K \in\{0,1\}^{k}$ and an input $x$ from the domain $S$ to return an output $y=F(K, x)$. The domain $S$ is for convenience $\{0,1\}^{*}$, or at least the set of all strings of length up to some very large maximum length. The notation $g \stackrel{R}{\leftarrow} F$ is shorthand for $K \stackrel{R}{\longleftarrow}$ $\{0,1\}^{k} ; g \leftarrow F(K, \cdot)$. We let $R$ denote the family of all functions of $S$ to $\{0,1\}^{l}$ so that $g \stackrel{R}{\leftarrow} R$ denotes the operation of selecting at random a function of $S$ to $\{0,1\}^{l}$. A distinguisher $D$ is an algorithm that takes an oracle for a function $g: S \rightarrow\{0,1\}^{l}$, and after computing with this oracle returns a bit. The following is the notion of [9] concretized as per [3]. 
Definition 2. Let $F, R$ be as above, let $D$ be a distinguisher, and suppose $t, q, \mu \geq 0$. Define the advantage of $D$, and the advantage function of $F$, respectively, as

$$
\begin{aligned}
\mathbf{A d v}_{F}^{\text {prf }}(D) & =\operatorname{Pr}\left[D^{g}=1: g \stackrel{R}{\leftarrow} F\right]-\operatorname{Pr}\left[D^{g}=1: g \stackrel{R}{\leftarrow} R\right] \\
\mathbf{A d v}_{F}^{\text {prf }}(t, q, \mu) & =\max \left\{\mathbf{A d v}_{F}^{\text {prf }}(D)\right\} .
\end{aligned}
$$

where the maximum is over all $D$ with time-complexity at most $t$, making at most $q$ oracle queries, these totalling at most $\mu$ bits.

ALL-OR-NOTHING TRANSFORMS. An all-or-nothing transform is an efficiently computable, keyless, randomized transformation AONT which maps a message to a sequence of blocks such that given the AONT of some message, one can easily compute the original message [12]. The (deterministic) inverse transformation permitting recovery of the message from the output is denoted $\mathrm{AONT}^{-1}$. Security pertains to the question of what information you can compute about the message if you are given all but one of the output blocks, and several notions have been suggested [12,68]. We provide our formalization and compare it to the others later.

We assume for simplicity that the AONT takes input messages of length $m$ and has outputs of length $s n$. The attack allowed is non-adaptive, meaning the adversary fixes beforehand the position of the output block that will be omitted. Denote this by $L \in\{1, \ldots, s\}$. During the find stage the adversary comes up with a pair of messages $M_{0}$ and $M_{1}$, both of length $m$. In its guess stage it is given a AONT for one of the plaintexts $M_{0}, M_{1}$, with block $L$ missing. The adversary wins if it correctly guesses which message goes with the challenge AONT. If $X \in\{0,1\}^{s n}$ is a string of $s$ blocks, each $n$-bits long, then we let $X[1, \ldots, L-1, L+1, \ldots, s]$ denote the string consisting of blocks $1, \ldots, L-1, L+1, \ldots, s$ of $X$, meaning all but block $L$.

Definition 3. Let AONT be a (randomized) algorithm taking an input of length $m$ and returning an output of length $s n$. Let $L \in\{1, \ldots, s\}$ be a block number. $S t$ denotes some state information. For $b=0,1$ define the experiment

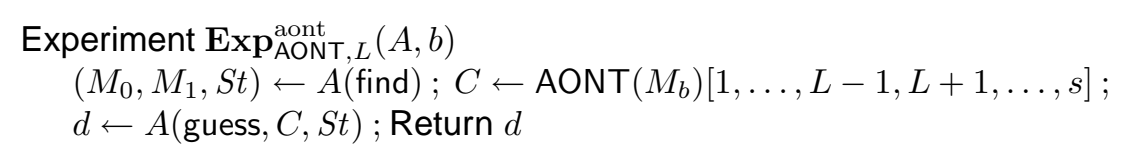

Now define the advantage of $A$ and the advantage function of AONT, respectively, as follows:

$$
\begin{aligned}
\mathbf{A d v}_{\mathrm{AONT}, L}^{\text {aont }}(A) & =\operatorname{Pr}\left[\mathbf{E x p}_{\mathrm{AONT}, L}^{\text {aont }}(A, 0)=0\right]-\operatorname{Pr}\left[\mathbf{E x p}_{\mathrm{AONT}, L}^{\text {aont }}(A, 1)=0\right] \\
\mathbf{A d v}_{\mathrm{AONT}, L}^{\text {aont }}(t) & =\max \left\{\mathbf{A d}_{\mathbf{A O N T}, L}^{\text {aont }}(A)\right\}
\end{aligned}
$$

where the maximum is over all $A$ with "time-complexity" $t$.

We now compare this to other notions, in all cases considering an adversary having a string $C$ consisting of all but one block of the output. Rivest [12] asks that given $C$ it be computationally infeasible to get any non-trivial information about any block of the message. Our definition is stronger than his, meaning any AONT secure in our 


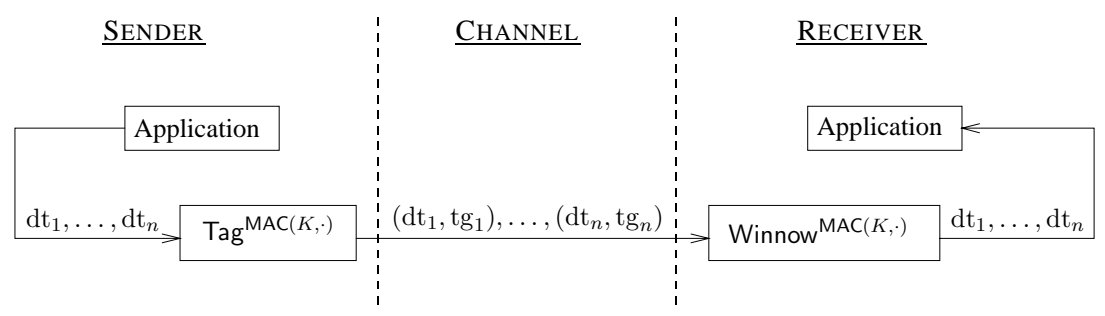

Fig. 1. Authentication channel

sense is secure in his sense. Boyko's definition of security [6] asks that given $C$ it be computationally infeasible to get any non-trivial information about the message as a whole, not just individual blocks. Desai [8] asks that $C$ be indistinguishable from a random string of the same length. Our definition is weaker than either of these, in the sense that any AONT secure in their sense is secure in our sense.

\section{Defining Chaffing and Winnowing}

Fix a map MAC: $\{0,1\}^{k} \times\{0,1\}^{*} \rightarrow\{0,1\}^{l}$ to be used as a message authentication code. (Security assessments will assume that this map is a pseudorandom function, but discussions and constructions will refer to it as a MAC.) A packet is a pair (dt, tg) consisting of data dt and a tag tg where the length of $\operatorname{tg}$ is $l$-bits, the length of the output of MAC. A packet (dt, tg) is valid with respect to $\mathrm{MAC}_{K}$ - where $K \in\{0,1\}^{k}$ is some key for the MAC - if $\mathrm{MAC}_{K}(\mathrm{dt})=\mathrm{tg}$, and invalid with respect to $\mathrm{MAC}_{K}$ otherwise. When $\mathrm{MAC}_{K}$ is understood, we simply talk of valid and invalid packets.

The sender and receiver have an authenticated channel of communication based on the MAC. Each party has a module responsible for authentication. These modules hold in common a key $K \in\{0,1\}^{k}$ for the MAC. When the sender wants to send data $\mathrm{dt}$ to the receiver in an authenticated way, the sender passes $\mathrm{dt}$ to its authentication module, which creates the (valid) packet $\operatorname{Pkt}=(\mathrm{dt}, \operatorname{MAC}(K, \mathrm{dt}))$. This packet is sent to the receiver. We call this the "tag" procedure. The packet is received by the receiver's authentication module, which verifies the tag. If the tag is valid, it passes the data "up" to the receiver. If the tag is not valid, the packet is simply discarded; nothing is passed up to the receiver. The receiving module thus acts as a "filter", separating "wheat" (valid) packets from "chaff" (invalid) packets, and passing to the receiver only the data from the valid packets. This is what [11] calls the "winnow" procedure. The two procedures are specified in detail below, and the channel is depicted in Figure 1

Definition 4. [MAC-based tag and winnow procedures] We associate to a MAC function MAC: $\{0,1\}^{k} \times\{0,1\}^{*} \rightarrow\{0,1\}^{l}$ the following tag and winnow procedures. The tag procedure produces a valid packet from the input data. The winnow procedure takes as input a stream of packets and returns the data of the valid packets: 


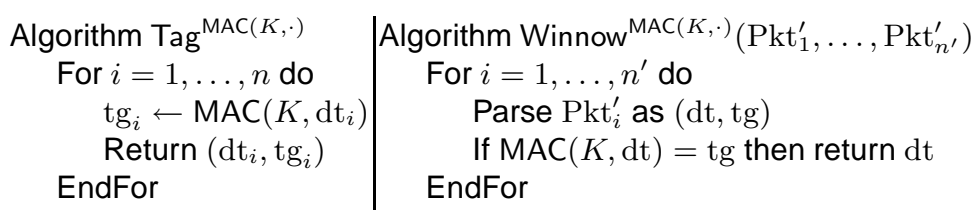

Here $K \in\{0,1\}^{k}$ is a key for the MAC and $n$ is the number of packets in the input stream.

The receiver has no direct access to the packets or their MACs, no access to (or even knowledge of) the invalid packets, which are simply discarded by the winnow procedure. The receiver only gets, in order, the data part of the valid packets.

Definition 5. [ATPT] An authentication to privacy transform (ATPT) with tag length $l$ is a triple ATPT $=$ (MakeWheat, AddChaff, Recover) of algorithms, where

- MakeWheat takes as input a message $M$ and returns a sequence $w_{1}, \ldots, w_{n}$ of strings called the wheat strings

- AddChaff takes as input a sequence $\mathrm{Pkt}_{1}, \ldots, \mathrm{Pkt}_{n}$ of packets called the wheat packets and returns another sequence $\mathrm{Pkt}_{1}^{\prime}, \ldots, \mathrm{Pkt}_{n^{\prime}}^{\prime}$ of packets

- Recover takes as input strings $w_{1}, \ldots, w_{n}$ and returns a message $M$.

The first two algorithms can be probabilistic or stateful (accessing a global state variable such as a counter). The last algorithm is usually deterministic and stateless.

An ATPT above is used in combination with an authentication channel to provide confidentiality. The way the process works is depicted and explained in Figure 2 Our interest is in the security of this entire procedure viewed as a symmetric encryption scheme. For this purpose it is convenient to think of it more as a standard symmetric encryption scheme, consisting of a key generation, encryption and decryption procedure. (The fact that it works by chaff and winnow is irrelevant to the security, although of course crucial to policy debate.) Below, we specify the symmetric encryption scheme that results from running a given ATPT over a given authentication channel, by specifying the three constituent algorithms.

Definition 6. Let ATPT $=$ (MakeWheat, AddChaff, Recover) be an ATPT with tag length $l$, and let MAC: $\{0,1\}^{k} \times\{0,1\}^{*} \rightarrow\{0,1\}^{l}$ be a MAC. Associated to them is a canonical encryption scheme $(\mathcal{K}, \mathcal{E}, \mathcal{D})$. The key generation algorithm $\mathcal{K}$ is the same as that of the MAC, namely it outputs a random $k$-bit key $K$, and the encryption and decryption algorithms are as follows:

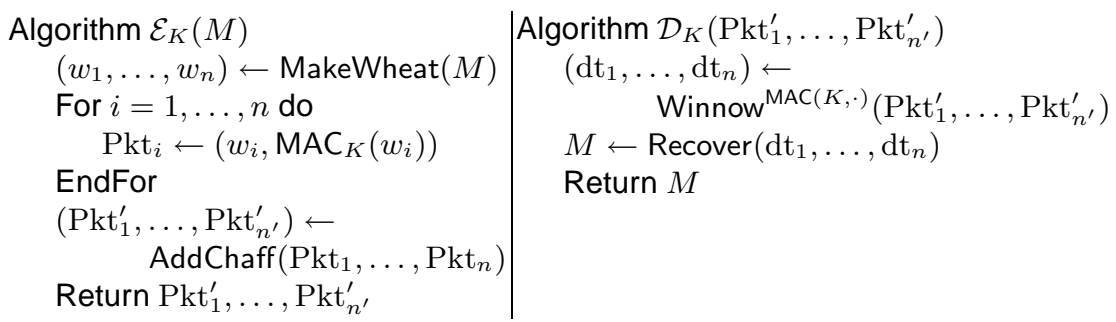

We require that $\mathcal{D}_{K}\left(\mathcal{E}_{K}(M)\right)=M$ for all $M \in\{0,1\}^{*}$. 


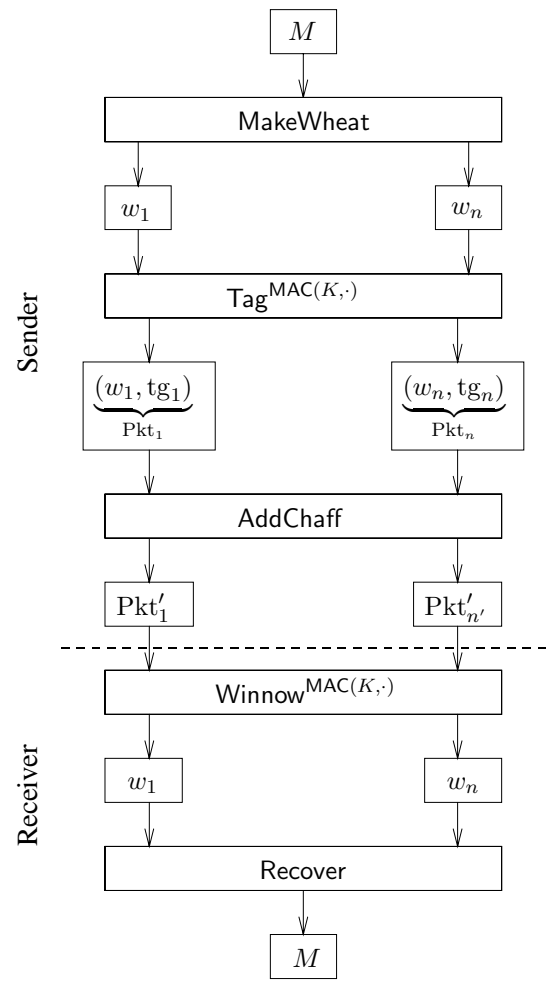

The (plaintext) message $M$ is first processed by a (keyless) transform MakeWheat to yield a sequence $w_{1}, \ldots, w_{n}$ of strings, each of which is MACed to yield a stream $\mathrm{Pkt}_{1}=$ $\left(w_{1}, \operatorname{tg}_{1}\right), \ldots, \mathrm{Pkt}_{n}=\left(w_{n}, \operatorname{tg}_{n}\right)$ of valid packets, where $\operatorname{tg}_{i}=\operatorname{MAC}\left(K, w_{i}\right)$ for $i=$ $1, \ldots, n$. The (keyless) AddChaff procedure adds chaff packets to produce a new stream $\mathrm{Pkt}_{1}^{\prime}, \ldots, \mathrm{Pkt}_{n^{\prime}}^{\prime}$ of packets (the ciphertext) which is sent to the receiver. They hit the receiver's winnow (cf. Definition 4) which discards packets with invalid MACs, and passes up to the receiver the data from the valid packets. A (keyless) Recover procedure now puts this data together to get back the original message $M$. The three keyless algorithms MakeWheat, AddChaff, and Recover comprise what we call the ATPT (authentication to privacy transform) - they enable the possibility of obtaining confidentiality via an existing authentication channel without the addition of any extra cryptographic elements.

Fig. 2. Chaff-and-winnow based "encryption".

The last requirement is made so that this is a valid symmetric encryption scheme, meaning correctly encrypted data can be decrypted by a receiver that knows the secret key.

In the sequel, we will specify chaff-and-winnow based encryption schemes directly as standard symmetric encryption schemes, because this is more conducive to security assessments. Accordingly it is useful to have the following terminology.

Definition 7. Let $\mathrm{SE}=(\mathcal{K}, \mathcal{E}, \mathcal{D})$ be a symmetric encryption scheme. We say that $\mathrm{SE}$ is a chaff-and-winnow based encryption scheme if there exists an ATPT transform ATPT and a MAC MAC: $\{0,1\}^{k} \times\{0,1\}^{*} \rightarrow\{0,1\}^{l}$ such that SE is exactly the canonical confidentiality procedure associated to ATPT and MAC as per Definition 6 .

\section{Analysis of Rivest's Schemes}

As above, MAC: $\{0,1\}^{k} \times\{0,1\}^{*} \rightarrow\{0,1\}^{l}$ is a message authentication code. In the bit-by-bit scheme, the sender maintains a counter $c t r$ that is initially zero. The encryption procedure (more precisely, the MakeWheat algorithm) increments this counter upon each invocation. Assume all messages to be encrypted have length $m$. 
Scheme 1. [Bit-by-bit CW] The key generation algorithm $\mathcal{K}$ of this symmetric encryption scheme returns a random $k$-bit key $K$ for the MAC, and the encryption and decryption algorithms are as follows:

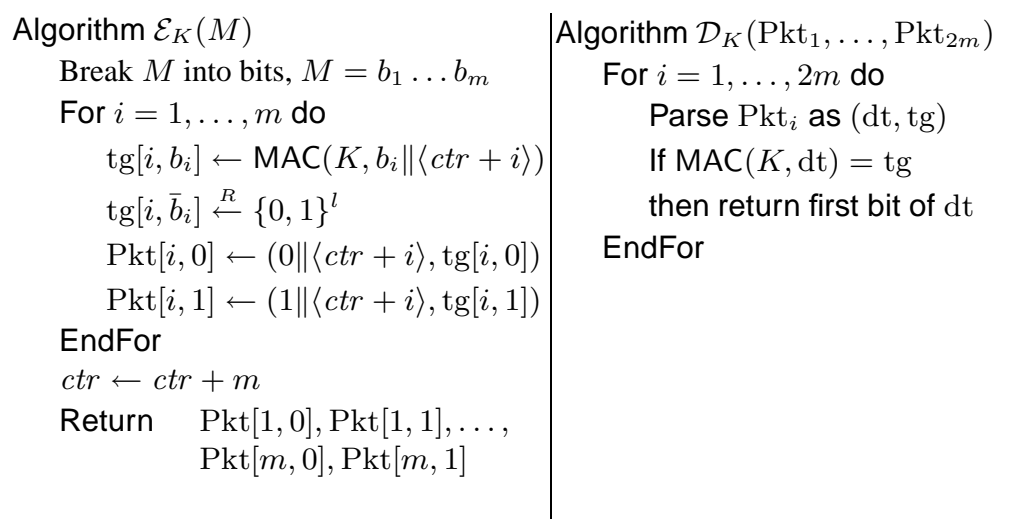

Here $\bar{b}$ denotes the complement bit of $b$ and $\langle i\rangle$ denotes the binary representation of integer $i$ as a binary string of some fixed, predefined length $p$. The "wheat" packets are $\operatorname{Pkt}\left[i, b_{i}\right]$ for $i=1, \ldots, m$ and the "chaff" packets are $\operatorname{Pkt}\left[i, \bar{b}_{i}\right]$ for $i=1, \ldots, m$.

In the full version of this paper [1] we show formally that the above (and other schemes of this paper) are chaff-and-winnow based encryption schemes by saying what are the algorithms MakeWheat, AddChaff, and Recover.

The following theorem shows that this scheme meets the "find-then-guess" notion of privacy under the assumption that MAC is a PRF. The reduction is almost tight. The proof is in [1].

Theorem 1. Let MAC: $\{0,1\}^{k} \times\{0,1\}^{*} \rightarrow\{0,1\}^{l}$ be a pseudorandom function and let $\mathrm{SE}=(\mathcal{K}, \mathcal{E}, \mathcal{D})$ be the bit-by-bit chaff-and-winnow based encryption scheme of Scheme $\square$. Assume the counter is $p$-bits long. Then for any $t, q, \mu$ with $\mu<2^{p}$

$$
\mathbf{A d v}_{\mathrm{SE}}^{\mathrm{priv}}(t, q, \mu) \leq 2 \cdot \mathbf{A d v}_{\mathrm{MAC}}^{\mathrm{prr}}\left(t, q^{\prime}, \mu^{\prime}\right)
$$

where $q^{\prime}=\mu$ and $\mu^{\prime}=(1+p) \mu$. I

If the counter is allowed to wrap around the scheme is obviously insecure. It is possible to use randomness instead of a counter. In this case each bit of the message is concatenated with random value represented as a string of some fixed predefined length. This value is drawn at random for each bit of the message. The analysis is analogous but the concrete security is worse due to birthday attacks.

The security of these schemes comes with a price. They are very inefficient since they have large data expansion: if the message is $m$ bits long then $2 m(1+p+l)$ bits are transmitted, where $p$ is the length of a counter and $l$ is the length of the output of the MAC. Bleichenbacher suggested that it is possible to reduce the communication cost by a factor of two by selecting at random and sending just one packet for each bit, either a chaff or a wheat packet. The receiver checks the validity of the data packet 
and complements the bit if the packet is invalid. Bleichenbacher further suggested that it is possible to reduce the communication even more if the sender authenticates each byte of the message and transmits only the computed MAC, but not the byte itself. The receiver then has to compute MACs for all possible bytes and take that for which the MACs match.

Another scheme mentioned in [11] authenticated message blocks of some length rather than single bits, but the author already indicated that it was insecure under stringent notions of privacy such as the one we use here. We go on to the scattering scheme.

As before let MAC: $\{0,1\}^{k} \times\{0,1\}^{*} \rightarrow\{0,1\}^{l}$ be our message authentication code. In the scattering scheme, the output of an AONT is viewed as a sequence of $s$ blocks, each $n$ bits long. The ciphertext will contain $s$ wheat packets interspaced with $s^{\prime}>0$ chaff packets, where $s^{\prime}$ is a parameter of the scheme. The wheat packet positions are a random subset of $\left\{1, \ldots, s+s^{\prime}\right\}$. The full description follows.

Scheme 2. [Scattering scheme] We fix an all-or-nothing transform AONT: $\{0,1\}^{m}$ $\rightarrow\{0,1\}^{s n}$. We assume that all messages to be encrypted have length $m$. The key generation algorithm $\mathcal{K}$ of this symmetric encryption scheme returns a random $k$-bit key $K$ for the MAC, and the encryption and decryption algorithms are as follows:

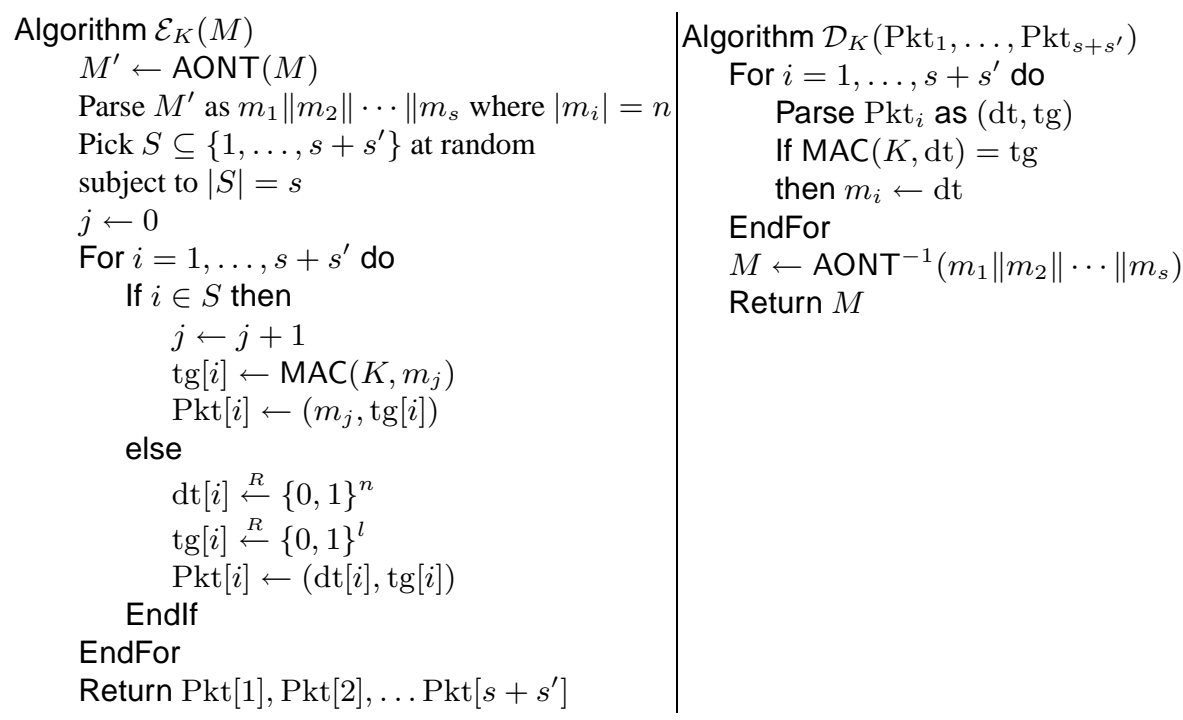

The most obvious attack is to test each group of $s$ packets to see whether they are the wheat packets. The adversary goes through all size $s$ subsets of the packets. In each case it forms a candidate output of AONT and applies AONT ${ }^{-1}$. Assuming it knows some partial information about the message, it can tell when it got the choice of the subset right. The time taken by this attack is proportional to $\left(\begin{array}{c}s+s^{\prime} \\ s\end{array}\right)$.

The intuition for security given in [11] is that this is the best possible attack. The complexity is large as long as both $s$ and $s^{\prime}$ are above some minimal threshold; for example, both more than 128 . Accordingly we could set $s^{\prime}=128$ and choose the AONT so that its output always had at least 128 blocks. 
A closer look reveals however that security is not so straightforward. For example, another thing to consider is the effect of equal data blocks. If the data blocks in two packets are equal, an adversary can get some information by looking at their tags: if the tags are unequal, they cannot both be wheat packets, because the MAC is deterministic. This can reduce the complexity of an attack, indicating that the time-complexity of an attack must also be a function of the block size $n$ of the output.

There are other such considerations, but more importantly, we claim that Rivest's notion of security for the AONT can be shown to be insufficient to make this scheme secure. An example illustrating this is to consider an AONT each of whose output blocks has the property that the first few bits are 0. (One can show that if any AONT meeting Rivest's definition exists, then so does one with this property.) But with this AONT, Scheme 2 can be broken because wheat packets can be distinguished from chaff packets: the wheat packets are the ones whose data has first few bits zero. The same counterexample shows that Definition 3 is also not enough.

The example AONT we constructed above does not however meet Boyko's stronger notion of security for AONTs [6], so the next question is whether Scheme 2] could be proven secure under this stronger notion. However even with this stronger notion it is unclear one can prove security. The reason is that the ciphertext contains the complete output of the AONT, while the security property of the AONT pertains to a setting where the adversary has no information about at least one block of the output of the AONT. This makes it unclear how to do a reduction. Indeed, the security property of an AONT does not seem to mesh well with what is required to prove security of Scheme 2 We will see next that a positive statement can be made by considering a particular AONT, namely the OAEP transform of Bellare and Rogaway [5]. But in general, as the transform used in the initial step, an AONT seems to be neither sufficient nor necessary for the security of Scheme2.

The OAEP transform appeals to random oracles $G:\{0,1\}^{n} \rightarrow\{0,1\}^{m}$ and $H$ : $\{0,1\}^{m} \rightarrow\{0,1\}^{n}$ where $n$ is the length of the OAEP seed and $m$ as usual is the message length. It takes as input an $m$-bit string $M$ and proceeds as follows-

$$
\begin{aligned}
& \text { Algorithm } \operatorname{OAEP}^{G, H}(M) \\
& \quad r \stackrel{R}{\leftarrow}\{0,1\}^{n} ; y \leftarrow G(r) \oplus M ; w \leftarrow H(y) \oplus r ; \text { Return } w \| y
\end{aligned}
$$

Boyko showed that OAEP is an AONT, but this will not help us here given the above discussion. Instead, we go back to the transform itself and prove the security of Scheme 2 when AONT is set to OAEP.

As with any proof concerning OAEP, we work in the random oracle model of [4]. We must "lift" our definitions to allow all algorithms and parties, including the adversary, access to the random oracles $G, H$. Briefly, modify $\operatorname{Exp}_{\mathrm{SE}}^{\text {priv }}(A, b)$ in Definition 1 to begin by picking $G, H$ randomly. Allow $\mathcal{E}_{K}$ and $A$ oracle access to $G, H$. Allow the scheme advantage to take extra parameters, $\operatorname{Adv}_{\mathrm{SE}}^{\text {priv }}\left(t, q, \mu ; q_{G}, q_{H}\right)$, these being bounds on the number of queries made by the adversary to the oracles in question.

The bound below reflects the above intuition: it is inversely proportional to $\left(\begin{array}{c}s+s^{\prime} \\ s\end{array}\right)$ and also to $2^{n}$. This shows that for OAEP the security is what one would have liked it to be for a "good" AONT. The proof of Theorem 2 can be found in [1]. 
Theorem 2. Let $n, m$ be integers with $m$ a multiple of $n$. Let MAC: $\{0,1\}^{k} \times\{0,1\}^{*} \rightarrow$ $\{0,1\}^{l}$ be a pseudorandom function. Let $\mathrm{SE}=(\mathcal{K}, \mathcal{E}, \mathcal{D})$ be Scheme 2 using OAEP as the AONT, with parameters $n, s, s^{\prime}$ where $s=m / n+1$. For any $t, q$ we let $\mu=n q(s-1)$. Assume $q_{H} \leq 2^{n} / 2$ and $q_{G} \leq(1 / 2) \cdot\left(\begin{array}{c}s+s^{\prime} \\ s\end{array}\right)$. Then

$$
\begin{aligned}
& \mathbf{A d v}_{\mathrm{SE}}^{\mathrm{priv}}\left(t, q, \mu ; q_{G}, q_{H}\right) \leq \\
& \frac{2 q_{H}}{\left(\begin{array}{c}
s+s^{\prime} \\
s-1
\end{array}\right)}+\frac{2 q_{G}+(q+1)^{2} \cdot\left[\left(s+s^{\prime}\right)^{2}+1\right]}{2^{n}}+\mathbf{A d v}_{\mathrm{MAC}}^{\mathrm{prf}}\left(t^{\prime}, q^{\prime}, \mu^{\prime}\right)
\end{aligned}
$$

where $t^{\prime}=t, q^{\prime}=q\left(s+s^{\prime}\right)$ and $\mu^{\prime}=n q\left(s+s^{\prime}\right)$.

\section{A New Chaffing-and-Winnowing Scheme}

Here we suggest an alternative scheme that has low data expansion and analyze its advantage function. It returns to much more "standard" paradigms of encryption than the scattering scheme. Simply apply an AONT to the message and then encrypt the first block of the message. If the last encryption is done by chaffing-and-winnowing, say using the bit-by-bit scheme, the whole scheme is also a chaffing-and-winnowing scheme, since the AONT is keyless. The savings in bandwidth comes from the fact that the number of bits encrypted using the bit-by-bit scheme is independent of the length of the message.

Scheme 3. Let AONT be an all-or-nothing transform taking input messages of length $m$ and returning outputs of length $s n$. The output is viewed as a sequence of $n$-bit blocks. Let se $=(\mathcal{K}$, e, d) be the bit-by-bit scheme of Scheme1 with message space $\{0,1\}^{n}$. The new scheme is $\mathrm{SE}=(\mathcal{K}, \mathcal{E}, \mathcal{D})$ where

$$
\begin{array}{c|c}
\text { Algorithm } \mathcal{E}_{K}(M) & \text { Algorithm } \mathcal{D}_{K}\left(C_{1} \|\left(m^{\prime \prime}, \tau\right)\right) \\
& M^{\prime} \leftarrow \text { AONT }(M) \\
\text { Let } m^{\prime} \text { be the first block of } M^{\prime} \text { and } m^{\prime \prime} \text { the rest } & \left.M^{\prime} \leftarrow C_{1}\right) \\
C_{1} \leftarrow m_{K}^{\prime \prime}\left(m^{\prime}\right) & M \leftarrow \mathrm{AONT}^{-1}\left(M^{\prime}\right) \\
\text { Return } C_{1} \|\left(m^{\prime \prime}, \operatorname{MAC}\left(K, m^{\prime \prime}\right)\right) & \text { Return } M
\end{array}
$$

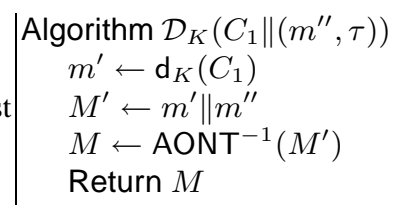

Note that the MAC attached to $m^{\prime \prime}$ is irrelevant to security; it is only there in order to make the final scheme a chaffing-and-winnowing scheme.

We now analyze the security of Scheme 3 Refer to Definition 3 for the definition of the advantage function of AONT and note that $L=1$ in this case, meaning we are requiring security only in the case where the first block is the one not provided to the adversary. A proof of the following theorem is in [1].

Theorem 3. Let MAC: $\{0,1\}^{k} \times\{0,1\}^{*} \rightarrow\{0,1\}^{l}$ be a pseudorandom function and let AONT be an all-or-nothing transform with input length $m$, output length sn. Let $\mathrm{SE}=(\mathcal{K}, \mathcal{E}, \mathcal{D})$ be Scheme 3 using AONT as the all-or-nothing transform and

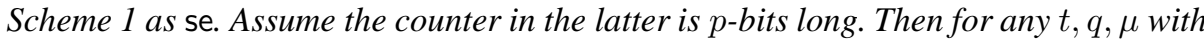
$\mu=q m$ and $q n<2^{p}-$ 


$$
\mathbf{A d v}_{\mathrm{SE}}^{\text {priv }}(t, q, \mu) \leq 2 \cdot \mathbf{A} \mathbf{d} \mathbf{v}_{\mathrm{MAC}}^{\mathrm{prr}}\left(t, q_{1}, \mu_{1}\right)+\mathbf{A d v}_{\mathrm{AONT}, 1}^{\text {aont }}(t)
$$

where $q_{1}=q(n+1)$ and $\mu_{1}=q n(s+p)$.

A concrete instantiation can be obtained by using OAEP in the role of the AONT. The security of this instantiation relies on the fact that OAEP is a secure AONT [6], and the concrete security can be obtained by combining the above with the results in [6]. (In that case we would have to lift all of the above to the random oracle model, but this is easily done.)

\section{Acknowledgments}

We thank Ron Rivest and Daniel Bleichenbacher for helpful discussions, and the anonymous referees for their constructive comments.

The authors are supported in part by a 1996 Packard Foundation Fellowship in Science and Engineering and NSF CAREER Award CCR-9624439.

\section{References}

1. M. Bellare And A. Boldyreva, "The security of chaffing and winnowing," Full version of this paper, available via http://www-cse.ucsd.edu/users/mihir.

2. M. Bellare, A. Desai, E. Jokipit AND P. Rogaway, "A concrete security treatment of symmetric encryption: Analysis of the DES modes of operation," Proceedings of the 38th Symposium on Foundations of Computer Science, IEEE, 1997.

3. M. Bellare, J. Kilian ANd P. Rogaway, "The security of cipher block chaining," Advances in Cryptology - Crypto '94, Lecture Notes in Computer Science Vol. 839, Y. Desmedt ed., Springer-Verlag, 1994.

4. M. Bellare AND P. RogawAY, "Random oracles are practical: a paradigm for designing efficient protocols," Proceedings of the 1st Annual Conference on Computer and Communications Security, ACM, 1993.

5. M. Bellare, P. Rogaway, "Optimal asymmetric encryption - How to encrypt with RSA," Advances in Cryptology - Eurocrypt '94, Lecture Notes in Computer Science Vol. 950, A. De Santis ed., Springer-Verlag, 1994.

6. V. BОYKO, "On the security properties of OAEP as an all-or-nothing transform,"Advances in Cryptology - Crypto '99, Lecture Notes in Computer Science Vol. 1666, M. Wiener ed., Springer-Verlag, 1999.

7. R. Canetti, Y. Dodis, S. Halevi, E. Kushilevitz, A. Sahai, "Exposure-resilient functions and all-or-nothing transforms," Advances in Cryptology - Eurocrypt '00, Lecture Notes in Computer Science Vol. 1807, B. Preneel ed., Springer-Verlag, 2000.

8. A. DESAI, "The security of all-or-nothing encryption: protecting against exhaustive key search," Advances in Cryptology - Crypto '00, Lecture Notes in Computer Science Vol. 1880, M. Bellare ed., Springer-Verlag, 2000.

9. O. Goldreich, S. Goldwasser and S. Micali, "How to construct random functions,"Journal of the ACM, Vol. 33, No. 4, 210-217, (1986).

10. S. Goldwasser And S. Micali, "Probabilistic encryption," Journal of Computer and System Science, Vol. 28, 1984, pp. 270-299.

11. R. Rivest, "Chaffing and winnowing: Confidentiality without encryption," http: //theory.lcs.mit.edu/ rivest/publications.html.

12. R. RIVEST, "all-or-nothing encryption and the package transform," Proceedings of the 4th Workshop on Fast Software Encryption, Lecture Notes in Computer Science Vol. 1267, Springer-Verlag, 1997. 